Utah State University

DigitalCommons@USU

\title{
On Some Aspects of the Decision to Conserve or Harvest Old Growth Forest
}

Amitrajeet A. Batabyal

Utah State University

Follow this and additional works at: https://digitalcommons.usu.edu/eri

\section{Recommended Citation}

Batabyal, Amitrajeet A., "On Some Aspects of the Decision to Conserve or Harvest Old Growth Forest" (1998). Economic Research Institute Study Papers. Paper 148.

https://digitalcommons.usu.edu/eri/148

This Article is brought to you for free and open access by the Economics and Finance at DigitalCommons@USU. It has been accepted for inclusion in Economic Research Institute Study Papers by an authorized administrator of DigitalCommons@USU. For more information, please contact digitalcommons@usu.edu.

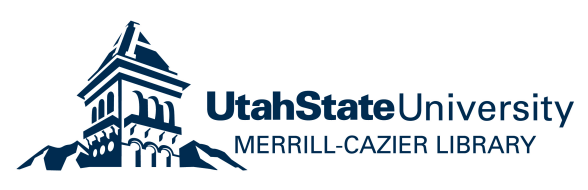


Economic Research Institute Study Paper

ERI \#98-10

\section{ON SOME ASPECTS OF THE DECISION TO CONSERVE OR HARVEST OLD GROWTH FOREST}

by

AMITRAJEET A. BATABYAL

Department of Economics

Utah State University

3530 Old Main Hill

Logan, UT 84322-3530

September 1998 


\title{
ON SOME ASPECTS OF THE DECISION TO CONSERVE OR HARVEST OLD GROWTH FOREST
}

\author{
Amitrajeet A. Batabyal, Associate Professor \\ Department of Economics \\ Utah State University \\ 3530 Old Main Hill \\ Logan, UT 84322-3530
}

The analyses and views reported in this paper are those of the author(s). They are not necessarily endorsed by the Department of Economics or by Utah State University.

Utah State University is committed to the policy that all persons shall have equal access to its programs and employment without regard to race, color, creed, religion, national origin, sex, age, marital status, disability, public assistance status, veteran status, or sexual orientation.

Information on other titles in this series may be obtained from: Department of Economics, Utah State University, 3530 Old Main Hill, Logan, Utah 84322-3530.

Copyright (C) 1998 by Amitrajeet A. Batabyal. All rights reserved. Readers may make verbatim copies of this document for noncommercial purposes by any means, provided that this copyright notice appears on all such copies. 


\title{
ON SOME ASPECTS OF THE DECISION TO CONSERVE OR HARVEST OLD GROWTH FOREST
}

\author{
Amitrajeet A. Batabyal
}

\begin{abstract}
In recent times, a great deal of concern has been expressed about the decline in the USA's old growth forests. Although there is broad agreement among reserachers that old growth forests provide timber and amenity services, and that forest harvesting decisions are inherently stochastic, there have been very few formal analyses that have explicitly accounted for these aspects of the old growth forest management problem. Consequently, in this paper I study these aspects of the problem by formulating the decision to conserve or harvest an old growth forest as a Markovian stochastic control problem. I then show how this control problem can be analyzed numerically using standard linear programming techniques.
\end{abstract}

Key words: linear programming, Markov chain, old growth forest 


\section{ON SOME ASPECTS OF THE DECISION TO CONSERVE OR HARVEST OLD GROWTH FOREST ${ }^{1}$}

\section{Introduction}

In recent times, a great deal of concern has been expressed about the decline in the USA's old growth forests. Environmentalists have alleged that the timber industry is responsible for the destruction of many pristine natural treasures that are old growth forests. In turn, the timber industry has pointed to the adverse region specific economic effects of a reduction in timber harvests (Tucker 1982; Lipske 1990; and Blumm 1994). Because old growth forests have a number of special characteristics and because it can take up to 250 years to develop old growth forests under natural conditions (Franklin et al. 1981), judicious management of old growth forests is now more important than ever before. There are three aspects of this management problem that deserve particular attention. First, as Reed (1993) and Reed and Ye (1994) noted, the management problem is inherently stochastic. Second, Hartman (1976), Bowes and Krutilla (1985), and Franklin and Forman (1987) all pointed out that old growth forests provide much more than just timber. In particular, these forests provide a number of amenity services that can generate quasioption value (Arrow and Fisher 1974; Hanemann 1989; and Batabyal 1996a). Third, owing to the analytical complexity of the management problem, forest managers typically use linear programming techniques to produce timber harvest schedules (Johnson and Scheurman 1977; Johnson, Jones, and Kent 1980).

${ }^{1}$ I thank two referees and Roy Haines-Young for their comments on a previous draft of this paper. I acknowledge funding from (i) the Faculty Research Grant program at Utah State University, and from (ii) the Utah Agricultural Experiment Station, Utah State University, Logan, UT 84322-4810, by way of grant UTA 024. Approved as UAES journal paper No. 4962. The usual disclaimer applies. 
While there are a few studies of the forest management problem in a stochastic context (see Clarke and Reed (1989), Reed (1993), and Batabyal (1996b)), these studies have typically avoided any real discussion of the numerical dimension of the problem. Similarly, a number of researchers (see Johnson and Scheurman 1977; and Berck and Bible 1984) addressed the numerical aspects of the problem. However, these researchers have not incorporated the effects of uncertainty into their analysis and nor have they analyzed the impact of the provision of amenity services from old growth forests. Given this state of affairs, it seems fair to say that there are very few studies of the old growth forest management problem that have accounted for all three aspects of the problem alluded to in the previous paragraph.

Consequently, in this paper I shall describe a Markov decision theoretic approach to the old growth forest management problem. This approach explicitly accounts for the fact that the management problem is inherently stochastic. I shall then focus on the numerical dimension of the problem by discussing how the relevant stochastic control problems can be solved using linear programming techniques. The rest of this paper is organized as follows. Section 2 outlines the essentials of the theoretical framework. Section 3 discusses the expected average net benefit criterion for forest management. Section 4 describes the expected discounted net benefit criterion for forest management. Section 5 offers concluding comments and discusses directions for future research.

\section{The Theoretical Framework}

My approach is related to earlier work by Dreyfus and Law (1977, pp. 172-87) and by Ross (1993, pp. 182-6). This approach can be viewed as a continuation of the approach discussed in 
Batabyal (1996b). Think of an old growth forest as a Markov process $\{X(t): t=0,1,2, \ldots$,$\} , which$ is observed at discrete points in time $t$ to be in any one of $S$ possible states. The states are numbered $1,2, \ldots, S$. After observing the state of the forest, a forest manager must take a particular action $a$. The two possible actions are to conserve $(a=C)$ or to harvest $(a=H)$. The set of all possible actions is denoted by $A=\{a=C, a=H\}$. Because the purpose of this paper is to focus on the conserve/harvest dichotomy, I have limited the forest manager's actions to two. However, the reader should note that the extension to a finite number of actions is straightforward. Consequently, there is no loss of generality involved in my current formulation. If the forest is in state $i$ at time $t$ and action $a$ is chosen, then the next state of the forest will be determined by the transition probability $P_{i j}(a)$. Because the forest is a Markov process, this transition probability depends only on the current state and the chosen action. Mathematically, we have $P\{X(t+1)=j / X(0), a(0), X(1)$, $a(1), \ldots, X(t)=i, a(t)=a\}=P_{i j}(a)$.

The forest manager's principal task is to choose an optimal management policy, i.e., a rule for choosing actions. In this paper, I shall constrain the manager's policy set to include stochastic Markovian policies only. These kinds of policies have two distinct features. First, in such policies, the action $a$ prescribed at time $t$ depends only on the state of the forest at that time. Second, the policy may call for an action to be chosen in accordance with a probability distribution function. In other words, a policy is a set of real numbers $\Theta=\left\{\theta_{i}(a), a \in A, i=1, \ldots, S\right\}$ with the meaning that if the forest is in state $i$, then action $a$ is chosen with probability $\theta_{i}(a)$. Given that $\theta_{i}(a)$ is a probability, we must have $\theta_{i}(a) \in[0,1], \forall i, a$ and $\Sigma_{\forall a} \theta_{i}(a)=1, \forall i$.

The reader should note that given a particular policy $\Theta$, the sequence of states $\{X(t): t=0,1, \ldots$,$\} constitutes a discrete time Markov chain with transition probabilities$ 
$P_{i j}(\Theta)=\Sigma_{\forall a} \theta_{i}(a) P_{i j}(a)$. Now suppose that for every possible policy $\Theta$, the discrete time Markov chain $\{X(t): t=0,1, \ldots\}$ is ergodic. Then I can let $\pi_{i a}$ be the stationary probability that the forest will be in state $i$ when action $a$ is chosen in accordance with policy $\Theta$. Mathematically, $\pi_{i a}=\lim _{t \rightarrow \infty} P_{\Theta}\{X(t)=i, a(t)=a\}$. With this probabilistic description of an old growth forest, I am now in a position to describe the forest manager's optimization problems under the two criteria mentioned in the last paragraph of section 1. I shall first consider the case in which the manager wishes to maximize the expected average net benefit accruing to society from the policy $\Theta$.

\section{The Expected Average Net Benefit Criterion}

In this case the forest manager does not discount the future net benefit resulting from the use of a particular policy $\Theta$. Suppose that when the forest is in state $i$, the manager chooses action $a$. Let the cost of undertaking this action be $C(i, a)$. Further, this action gives rise to social benefit $B(i, a)$. Hence, the net benefit to society from undertaking action $a$ in state $i$ is $N(i, a) \equiv B(\bullet, \bullet)-C(\bullet, \bullet)$. Because of the Markovian structure of the forest described in section 2, $N\{X(i), a(i)\}$ also denotes the net benefit accruing to society at time $i$. I can now define the expected average net benefit accruing to society per unit time when policy $\Theta$ is employed. This is given by

$$
\lim _{t \rightarrow \infty} E_{\Theta}\left[\frac{\sum_{i=1}^{i=t} N\{X(i), a(i)\}}{t}\right] .
$$

Using the probabilistic structure of the forest described in section 2, equation (1) can be simplified. First note that the stationary expected net benefit at time $t$ is $\lim _{t \rightarrow \infty} E[N\{X(t), a(t)\}]=\Sigma_{\forall i} \Sigma_{\forall a} \pi_{i a} N(i, a)$. This last expression tells us that the expected average net benefit accruing to society from the use of policy $\Theta$ is 


$$
\Sigma_{\forall i} \Sigma_{\forall a} \pi_{i a} N(i, a)
$$

Equation (2) denotes the forest manager's objective function and it is this expression that the manager wishes to maximize. The reader will note that this is a rather general formulation. In this formulation, the net benefit function $N(\bullet, \bullet)$ is an arbitrary function of the timber and the amenity services provided by the old growth forest. As a result, in this formulation it is possible to capture the different ways in which the timber and the nontimber services from old growth forests may affect the net benefit accruing to society.

Having outlined the forest manager's objective, I can now describe a minimum set of constraints that a well-posed maximization problem with the objective in equation (2) must satisfy. These constraints pertain to the stationary probability vector $\Pi=\left\{\pi_{i a}\right\}$. Because the $\pi_{i a}$ are the stationary probabilities for the forest under study, the first constraint simply requires that these probabilities be nonnegative. In other words, $\pi_{i a} \geq 0, \forall i, a$ must hold. The second constraint requires that these stationary probabilities sum to one when the summation is performed over all possible states and actions. In other words, $\Sigma_{\forall i} \Sigma_{\forall a} \pi_{i a}=1$ must hold. Finally, the third constraint stems from a defining equation which characterizes the stationary probabilities for discrete time Markov chains (see Wolff 1989, pp. 150-1). This constraint tells us that the stationary probability of being in some state $j$ must equal that same probability when that probability is computed by conditioning on the state and the action chosen one stage earlier in the evolution of the forest. In other words, $\Sigma_{\forall a} \pi_{j a}=\Sigma_{\forall i} \Sigma_{\forall a} \pi_{i a} P_{i j}(a), \forall j$ must hold.

Having specified the three constraints that the stationary probability vector $\Pi=\left\{\pi_{i a}\right\}$ must satisfy, I can now state the forest manager's dynamic programming problem completely. This manager solves 


$$
\max _{\Pi=\left\{\pi_{i a}\right\}} \Sigma_{\forall i} \Sigma_{\forall a} \pi_{i a} N(i, a)
$$

subject to

$$
\begin{gathered}
\pi_{i a} \geq 0, \forall i, a, \\
\Sigma_{\forall i} \Sigma_{\forall a} \pi_{i a}=1,
\end{gathered}
$$

and

$$
\Sigma_{\forall a} \pi_{j a}=\Sigma_{\forall i} \Sigma_{\forall a} \pi_{i a} P_{i j}(a), \forall j .
$$

The reader should note that equations (4)-(6) are the minimum necessary constraints in order for the above maximization problem to be well posed. In any specific instance, a forest manager may well have additional area constraints, harvest flow constraints, and/or harvest fluctuation constraints. The important thing to note is that the dynamic programming problem described by equations (3)-(6) is a linear programming problem. This is because the objective function and all the three constraints are linear functions of the decision variables, i.e., the $\pi_{i a}$. As Johnson and Scheurman (1977) and Berck and Bible (1984) noted, the additional constraints referred to above can generally also be expressed in a linear form. Hence, with some possible changes, this linear programming problem can be solved numerically by variants on methods such as the U.S. Forest Service harvest scheduling procedure FORPLAN.

Suppose that $\Pi^{*}=\left\{\pi_{i a}^{*}\right\}$ solves the problem described by equations (3)-(6). Then the forest manager's optimal policy is given by $\Theta^{*}$ where $\theta_{i}^{*}(a)=\pi_{i a}^{*} / \Sigma_{\forall a} \pi_{i a}^{*}$. In other words, in an optimal policy, the probability of choosing action $a$ in state $i$ is given by the ratio of the stationary probability of being in state $i$ when action $a$ is chosen to the stationary probability of being in state $i$. As Ross (1983, p. $103 ; 1993$, p. 185$)$ noted, in this kind of problem, the optimal policy $\Theta^{*}$ is typically 
nonstochastic. In other words, for every state $i$, the optimal conserve or harvest action to be taken by the forest manager is a deterministic function of the state $i$.

Thus far, we have seen that the modeling approach to the old growth forest management problem described in this section has two rather attractive features. First, we have seen that although the manager's optimization problem is really a dynamic programming problem, this problem is also a linear program. Consequently, in principle this problem can be solved by standard linear programming techniques. Second, despite the seemingly involved nature of the problem formulation, the manager's optimal policy is a rather simple one. In particular, this simple policy maps the state of the forest to the appropriate conserve or harvest action in a deterministic manner.

I now consider the case in which the manager discounts the future net benefit accruing to society from optimal forest management.

\section{The Expected Discounted Net Benefit Criterion}

Suppose that the discount rate is given by $\beta$, where $\beta \in(0,1)$. In this section, I shall suppose that the forest manager discounts the net benefit from management received at time $t$ at the rate $\beta^{t}$. In other words, the manager wishes to choose a policy $\Theta$ so as to optimize the expected discounted net benefit accruing to society per unit time. Mathematically, this amounts to maximizing

$$
E_{\Theta}\left[\sum_{i=0}^{i=\infty} \beta^{i} N\{X(i), a(i)\}\right] .
$$

The manager's objective in equation (7) can be simplified by defining $w_{j a}$ to be the expected discounted time that the forest is in state $j$ when action $a$ has been chosen. Now the expected discounted net benefit accruing to society per unit time under policy $\Theta$ is

$$
\Sigma_{\forall j} \Sigma_{\forall a} w_{j a} N(j, a)
$$


Once again, the reader will note the generality of this formulation. The net benefit function $N(\bullet, \bullet)$ captures the timber and the amenity services provided by the old growth forest in question in a very general way.

In order for the forest manager's optimization problem to be well posed, the $W=\left\{w_{j a}\right\}$ must satisfy three conditions. In turn, these conditions will form the three constraints on the manager's optimization problem. The first constraint simply requires the nonnegativity of the expected discounted time that the forest is in state $j$ when action $a$ is chosen. That is, we require $w_{j a} \geq 0, \forall j, a$ to hold. The last two constraints are more involved. In order to derive these constraints, I shall suppose that the initial state of the forest has been chosen according to the probabilities $d_{j}$. Put differently, $P\{X(0)=j\}=d_{j}, j=1, \ldots, t$. I now need a condition that will be satisfied by the expected discounted time in any state, $\Sigma_{\forall j} \Sigma_{\forall a} w_{j a}$, when policy $\Theta$ is chosen to manage the forest. This condition is contained in

Proposition 1: $\Sigma_{\forall j} \Sigma_{\forall a} w_{j a}=1 /(1-\beta)$.

Proof: For any event $E$, define the indicator variable $I_{E}$ by $I_{E}=1$ if $E$ occurs and $I_{E}=0$ otherwise. Then $w_{j a}=E_{\Theta}\left[\Sigma_{t=0}^{t=\infty} \beta^{t} I_{X(t)=j, a(t)=a}\right]$. Now $\Sigma_{\forall a} w_{j a}=\Sigma_{\forall a} E_{\Theta}\left[\Sigma_{\forall t} \beta^{t} I_{X(t)=j, a(t)=a}\right]=E_{\Theta}\left[\Sigma_{\forall t} \beta^{t} I_{X(t)=j}\right]$. Given this last expression, I get $\Sigma_{\forall j} \Sigma_{\forall a} w_{j a}=\Sigma_{\forall j} E_{\Theta}\left[\Sigma_{\forall t} \beta^{t} I_{X(t)=j}\right]=E_{\Theta}\left[\Sigma_{\forall t} \beta^{t}\right]=1 /(1-\beta)$.

Proposition 1 gives us the second constraint on the forest manager's optimization problem. This constraint tells us that the expected discounted time that the forest spends in any state $j$ is constant. This constant equals the reciprocal of one less $\beta$, the rate at which the manager discounts the future net benefit from policy $\Theta$. 
The final constraint on the manager's optimization problem involves a condition that must be satisfied by $\Sigma_{\forall a} w_{j a}$, the expected discounted time that the forest is in state $j$ under policy $\Theta$. This condition is given by

Proposition 2: $\Sigma_{\forall a} w_{j a}=d_{j}+\beta \Sigma_{\forall i} \Sigma_{\forall a} w_{i a} P_{i j}(a)$.

Proof: Once again for any event $E$, define the indicator variable $I_{E}$ by $I_{E}=1$ if $E$ occurs and $I_{E}=0$ otherwise. Now $\Sigma_{\forall a} w_{j a}=d_{j}+E_{\Theta}\left[\Sigma_{t=1}^{t=\infty} \beta^{t} I_{X(t)=j}\right]=d_{j}+\Sigma_{t=0}^{t=\infty} \beta^{t+1} \Sigma_{\forall i} \Sigma_{\forall a} E_{\Theta}\left[I_{X(t)=i, a(t)=a}\right] P_{i j}(a)$. This last e x p r e s s i n c a n b e f u r the r s i mplifie d to $d_{j}+\beta \Sigma_{\forall i} \Sigma_{\forall a} \Sigma_{\forall t} \beta^{t} E_{\Theta}\left[I_{X(t)=i, a(t)=a}\right] P_{i j}(a)=d_{j}+\beta \Sigma_{\forall i} \Sigma_{\forall a} w_{i a} P_{i j}(a)$.

Proposition 2 tells us that the expected discounted time that the forest is in state $j$ under policy $\Theta$ must equal that same time when that time is computed by conditioning on the state of the forest and the action chosen one stage earlier and adding to it the initial probability $d_{j}$.

From Propositions 1 and 2 it should be clear that we can think of the $W=\left\{w_{j a}\right\}$ as the expected discounted time that the forest is in state $j$ and action $a$ is chosen, given that the initial state is chosen according to the probabilities $d_{j}$. I can now state the forest manager's dynamic programming problem fully. This manager solves

$$
\max _{W=\left\{w_{j a}\right\}} \Sigma_{\forall j} \Sigma_{\forall a} w_{j a} N(j, a)
$$

subject to

$$
w_{j a} \geq 0, \forall j, a
$$

and

$$
\begin{gathered}
\Sigma_{\forall a} w_{j a}=d_{j}+\beta \Sigma_{\forall i} \Sigma_{\forall a} w_{i a} P_{i j}(a), \forall j \\
\Sigma_{\forall j} \Sigma_{\forall a} w_{j a}=1 /(1-\beta),
\end{gathered}
$$


The reader is reminded that equations (10)-(12) constitute a minimum set of constraints that a well-posed forest management problem must satisfy. In any specific context, additional constraints of the sort discussed in section 3 may well be necessary. However, the important point to note is that the dynamic programming problem described by equations (9)-(12) is a linear programming problem. This follows from the fact that in this problem, the objective function and the three constraints are all linear functions of the choice variables, i.e., the $w_{j a}$. Hence, with some variations, this problem can be solved numerically by linear programming techniques based on the simplex algorithm.

Let $W^{*}=\left\{w_{j a}^{*}\right\}$ be the solution to the optimization problem described by equations (9)-(12). Then the forest manager's optimal policy is given by $\Theta^{*}$ with $\theta_{j}^{*}(a)=w_{j a}^{*} / \Sigma_{\forall a} w_{j a}^{*}$. This tells us that the forest manager's optimal policy calls for choosing action $a$ in state $j$ with probability $\theta_{j}^{*}(a)$. In turn, this probability is given by the ratio of the expected discounted time that the forest is in state $j$ when action $a$ is taken to the expected discounted time that the forest is in state $j$ under every possible action. Like in section 3, the forest manager's optimal policy is typically nonrandomized (Hillier and Lieberman 1980, p. 569). This means that for every state $j$, the optimal conserve or harvest decision is a deterministic function of the state.

We have seen that with the expected discounted net benefit criterion, we can pose the forest manager's optimization problem meaningfully by replacing the concept of a stationary probability with the related notion of the expected discounted time that the forest spends in a particular state. The use of this notion enables us to pose the relevant dynamic programming problem as a linear programming problem. Finally, the optimal solution to this linear program typically calls for choosing actions that are a deterministic function of the current state. 
The practical significance of these results is twofold. First, the managerial function is simplified because these results tell us that a forest manager's optimal conserve/harvest decision depends only on the current state of the forest. This means that it is not necessary for a forest manager to know the manner in which the current state was reached. Second, these results also reduce the informational burden associated with the task of optimal forest management. Put differently, the forest manager need not worry about the specifics of any probability distribution function. This is because the manager's optimal policy does not require him to choose actions in accordance with some probability distribution function. Additional details on the kinds of dynamic programming problems that I have discussed in this paper can be found in Hillier and Lieberman (1980, pp. 548-87), in Denardo (1982, pp. 157-86) and in Ross (1983, pp. 29-48).

\section{Conclusions}

In this paper I presented two models of the old growth forest management problem within the context of a Markov decision theoretic framework. Let me now comment on the ways in which this framework addresses the three aspects of the forest management problem that I alluded to in the first paragraph of this paper. First, because this framework blends the theory of Markov chains with the theory of dynamic programming, I was able to formulate the forest manager's decision problem as two stochastic dynamic programming problems. Second, as per Franklin and Forman's (1987) suggestion, I was able to model the amenity services provided to society by old growth forests. Third, because the objective functions and the constraints to the two problems are linear in the decision variables, I noted that these problems are actually linear programs. This third point is important because as Denardo (1982, p. 162) has noted, not all sequential decision making problems 
can be posed as linear programming problems. Moreover, the fact that the forest management problems of this paper can be studied as linear programs means that variants of the simplex algorithm can be used to solve these problems.

The modeling approach of this paper has the advantage that the forest manager's optimal policy can typically be characterized by a deterministic function of the state even though the management problem is explicitly stochastic. From a practical standpoint, this considerably simplifies what otherwise might appear to look like a rather complicated management problem.

The research presented in this paper can be extended in a number of different directions. In what follows, I suggest two such directions. The first extension would be to analyze the old growth forest management problem when the manager uses policies of a non-Markovian character. An analysis of the effects of such policies will enable us to comprehend, inter alia, the role of history on current and future management policies. Second, in order to be directly useful for public policy, it will be necessary to determine ways in which extant numerical techniques for solving linear programs have to be modified to fit the attributes of the two linear programs discussed in this paper. Given the increasing importance of old growth forests to environmentalists and to the timber industry, one may look forward to significant new research developments in the future concerning the optimal management of this natural resource. 


\section{References}

Arrow, K. J., and A. C. Fisher. 1974. Environmental Preservation, Uncertainty, and Irreversibility. Quarterly Journal of Economics 88:312-9.

Batabyal, A. A. 1996a. The Timing of Land Development: An Invariance Result. American Journal of Agricultural Economics 78:1092-7.

Batabyal, A. A. 1996b. On Some Aspects of the Management of a Stochastically Developing Forest. Ecological Modelling 89:67-72.

Berck, P., and T. Bible. 1984. Solving and Interpreting Large-Scale Harvest Scheduling Problems by Duality and Decomposition. Forest Science 30:173-82.

Blumm, M. C. 1994. Public Choice Theory and the Public Lands: Why "Multiple Use" Failed. Harvard Environmental Law Review 18:405-32.

Bowes, M. D., and J. V. Krutilla. 1985. Multiple Use Management of Public Forestlands. In A. V. Kneese and J. L. Sweeney (eds.), Handbook of Natural Resource and Energy Economics, Vol. II, Amsterdam, The Netherlands: North-Holland.

Clarke, H. R., and W. J. Reed. 1989. The Tree-Cutting Problem in a Stochastic Environment. Journal of Economic Dynamics and Control 13:569-96.

Denardo, E. V. 1982. Dynamic Programming: Models and Applications. Englewood Cliffs, NJ: Prentice-Hall.

Dreyfus S. E., A. M. Law, and A. M. 1977. The Art and Theory of Dynamic Programming. New York, NY: Academic Press.

Franklin, J. F., K. Cromack, W. Denison, A. McKee, C. Maser, J. Sedell, F. Swanson, and G. Juday. 1981. Ecological Characteristics of Old-Growth Douglas-Fir Forests. USDA Forest Service General Technical Report PNW-118, Portland, OR.

Franklin, J. F., and R. T. Forman. 1987. Creating Landscape Patterns by Forest Cutting: Ecological Consequences and Principles. Landscape Ecology 1:5-18.

Hanemann, W. M. 1989. Information and the Concept of Option Value. Journal of Environmental Economics and Management 16:23-37.

Hartman, R. 1976. The Harvesting Decision when a Standing Forest Has Value. Economic Inquiry 14:52-8. 
Hillier, F. S., and G. J. Lieberman. 1980. Introduction to Operations Research, 3rd ed. San Francisco, CA: Holden-Day.

Johnson, K. N., and H. L. Scheurman. 1977. Techniques for Prescribing Optimal Timber Harvest. Forest Science Monograph \# 18.

Johnson, K. N., D. B. Jones, D. B., and B. M. Kent. 1980. A User's Guide to the Forest Planning Model (FORPLAN). Land Management Planning, USDA Forest Service, Fort Collins, CO.

Lipske, M. 1990. Who Runs America's Forests. National Wildlife 28:24-8.

Reed, W. J. 1993. The Decision to Conserve or Harvest Old-Growth Forest. Ecological Economics 8:45-69.

Reed, W. J., and J. J. Ye. 1994. The Role of Stochastic Monotonicity in the Decision to Conserve or Harvest Old Growth Forest. Natural Resource Modeling 8:47-80.

Ross, S. M. 1983. Introduction to Stochastic Dynamic Programming. San Diego, CA: Academic Press.

Ross, S. M. 1993. Introduction to Probability Models, 5th ed. San Diego, CA: Academic Press.

Tucker, W. 1982. Progress and Privilege: America in the Age of Environmentalism. Garden City, NY: Anchor Press/Doubleday.

Wolff, R. W. 1989. Stochastic Modeling and the Theory of Queues. Englewood Cliffs, NJ: Prentice-Hall. 